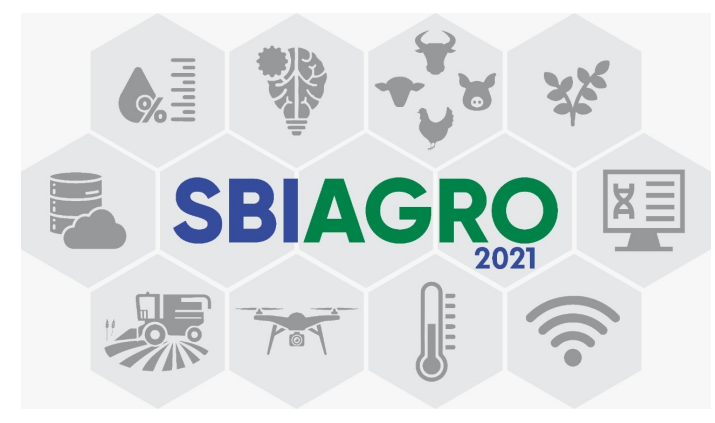

\section{Uma plataforma de dados geoespaciais para caracterização e combate à desertificação da Caatinga}

\author{
Jaudete Daltio, André Rodrigo Farias, \\ Lucíola Alves Magalhães, Daniela Maciel Pinto
}

\author{
${ }^{1}$ Empresa Brasileira de Pesquisa Agropecuária (Embrapa) \\ Soldado Passarinho 303, Fazenda Chapadão - Campinas - SP - Brasil \\ \{jaudete.daltio, andre.farias, \\ luciola.magalhaes, daniela.maciel\}@embrapa.br
}

\begin{abstract}
Desertification is the land-degradation process that occurs in arid, semi-arid, and dry sub-humid zones as a result of the joint action of natural source and anthropic factors. In Brazil, most of the susceptible areas are located in the Caatinga biome. This paper presents a data platform for characterizing and combating desertification that combines two main aspects: a collection of technologies to prevent, stem, or reverse desertification and a set of features that provides an overview of the regional agriculture trends. We expect to support technological disclosure strategies in suitable contexts and territories, and thus contribute to promoting sustainable agricultural development.
\end{abstract}

Resumo. Desertificação é o processo de degradação das terras que ocorre nas zonas áridas, semiáridas e sub-úmidas secas, resultado da ação conjunta de fatores de origem natural e antrópica. No Brasil, grande parte das áreas suscetíveis localiza-se no bioma Caatinga. Este artigo apresenta uma plataforma de dados para caracterização e combate à desertificação que integra dois aspectos principais: um acervo de tecnologias para prevenir e mitigar a desertificação e um conjunto de variáveis que provê uma visão geral das tendências da agropecuária regional. Espera-se subsidiar estratégias de disseminação tecnológica em contextos e territoriais adequados, contribuindo com a promoção do desenvolvimento agropecuário sustentável.

\section{Introdução e Motivação}

De acordo com a Convenção das Nações Unidas de Combate à Desertificação, desertificação é o processo de degradação das terras que ocorre nas zonas áridas, semiáridas e sub-úmidas secas, resultado da ação conjunta de múltiplos fatores que vão 
desde características naturais (principalmente solo, clima e relevo) a intervenções humanas no ambiente [UNCCD 1994]. Dada sua relevância, o combate à desertificação é um dos temas no Objetivo de Desenvolvimento Sustentável (ODS 15) ${ }^{1}$. O item 15.3 estabelece a meta de, até 2030, combater a desertificação, restaurar a terra e o solo degradado e lutar para alcançar um mundo neutro em termos de degradação do solo.

O conceito de Neutralidade da Degradação da Terra (Land Degradation Neutrality - LDN), destacado na definição da meta 15.3, refere-se a um estágio em que os recursos da terra encontram-se em um nível suficiente para a manutenção de todas as funções ecossistêmicas e da segurança alimentar das populações, em diferentes escalas temporais e espaciais [UNCCD 2019]. A neutralidade, segundo essa abordagem, se manifesta pela ausência de perda líquida no balanço de terras em processo de degradação e não degradadas. Assim, qualquer aumento de degradação deve ser necessariamente acompanhado por um crescimento proporcional de redução ou mitigação nessa ou em outras áreas, de forma que as perdas sejam compensadas pelos ganhos.

Associada a essa definição, desenvolveu-se uma estrutura científica para planejamento, implementação e monitoramento da LDN em diferentes contextos e sua instituição como uma meta global, na qual os países foram convidados a se comprometer voluntariamente com a LDN em nível nacional [Cowie et al. 2018]. Esse compromisso foi firmado, de forma institucional, a partir da adesão à Estratégia de Neutralidade da Degradação da Terra, mecanismo global de avaliação e implementação da LDN. Atualmente, esse instrumento reúne 128 países, conjunto do qual o Brasil faz parte.

Sob esta perspectiva, diversas iniciativas internacionais têm sido realizadas para atender aos objetivos estabelecidos pela LDN, principalmente no fomento a criação e difusão de tecnologias e práticas associadas à Gestão Sustentável da Terra (Sustainable Land Management - SLM). A SLM, por sua vez, define-se como o uso dos recursos da terra, incluindo solos, água, animais e plantas, visando a produção de bens para atender às necessidades humanas em constante mudança, onde há a garantia de manutenção simultânea de sua produtividade de longo prazo, a preservação de seus recursos e a manutenção de suas funções ambientais [Critchley et al. 2021].

Em âmbito nacional, iniciativas relacionadas ao tema são coordenadas pelo Ministério do Meio Ambiente (MMA), por meio do Programa de Ação Nacional de Combate à Desertificação e Mitigação dos Efeitos da Seca [PAN-Brasil 2004] e da Política Nacional de Combate à Desertificação (Lei no 13.153 de 30 de julho de 2015). Nesse sentido, propõe-se uma série de estratégias voltadas ao atendimento da neutralidade da terra que envolvem: oferta e disponibilização de tecnologias; proposição de sistemas integrados de produção sustentável e adequados às características locais; ampliação das possibilidades de agregação de valor e de processamento de produtos agropecuários de âmbito regional; capacitação e iniciativas de extensão rural.

Uma cooperação institucional entre a Embrapa e o MMA foi estabelecida em 2019 com o objetivo de auxiliar a atualização da estratégia ministerial de combate à desertificação no bioma Caatinga e a implementação da LDN. A adoção da Caatinga como recorte territorial para essa iniciativa justifica-se por suas características únicas em termos fitofisionomias e biodiversidade, a existência de complexos arranjos sociais e econômicos

\footnotetext{
${ }^{1}$ https://brasil.un.org/pt-br/sdgs/15
} 
em âmbito regional e por reunir parcela importante de áreas suscetíveis a processos de desertificação.

A proposta da cooperação entre a Embrapa e o MMA tem dois pilares: (1) a caracterização das dinâmicas territoriais no bioma, principalmente associadas à produção agropecuária e as áreas suscetíveis à desertificação e; (2) a sistematização de soluções tecnológicas aderentes ao tema e relevantes para as estratégias de neutralidade da terra. Estes dois pilares foram integrados na Plataforma de Informações para Combate à Desertificação da Caatinga, elaborada para materializar os resultados desta cooperação. O objetivo deste artigo é descrever esta plataforma.

Analisar as características territoriais tem se mostrado um importante instrumento para iniciativas de promoção de inovação, a adoção de tecnologias e disseminação de informações [da Silva et al. 2018]. Espera-se que os resultados deste trabalho contribuam para o diagnóstico qualificado das cadeias produtivas da Caatinga e possam oferecer aos produtores rurais, extensionistas, gestores públicos e privados um canal para divulgar e disseminar as soluções tecnológicas disponíveis para a sua região.

\section{Bases de Dados da Plataforma}

\subsection{Tecnologias da Embrapa}

Além de referência na produção agrícola mundial, o Brasil se destaca entre os que mais geram e publicam conhecimento em ciências agrárias. A Empresa Brasileira de Pesquisa Agropecuária (Embrapa) tem uma contribuição significativa na pesquisa agrária nacional. Ao longo de sua existência, a Embrapa registra mais de 270 mil publicações técnicocientíficas e 1.860 soluções tecnológicas aptas a serem transferidas e aplicadas pelo setor produtivo, disponíveis para acesso público ${ }^{2}$.

Apesar do grande volume de informações e tecnologias disponíveis, atingir diferentes públicos-alvo é um constante desafio. Há uma carência de dados organizados, sistematizadas e disponíveis em sistemas de informação e comunicação que cheguem efetivamente aos produtores rurais [Penteado-Filho 2010]. Além disso, a indexação deste conteúdo no âmbito corporativo e, consequentemente, seus mecanismos de busca, não contempla aspectos territoriais e nichos de atuação. Muitas tecnologias lançadas pela Embrapa associadas de forma direta ou indireta aos temas prevenção à desertificação, degradação da terra e recuperação de áreas por manejo florestal ou manejo integrado, se encontram dispersas em diversas categorias de soluções.

Para o desenvolvimento da plataforma descrita neste trabalho, realizou-se de uma seleção inicial de tecnologias pertinentes ao tema a partir da combinação de filtros de busca (por exemplo, desertificação, caatinga, degradação). Adotou-se, ainda, os procedimentos registrados por [Pinto et al. 2019]. A seleção inicial foi refinada a respeito de sua aderência ao conceito de desertificação (controle de processos erosivos, tecnologias de conservação da água e solo) e práticas sustentáveis. A partir desta seleção, pesquisadores de 27 diferentes centros de pesquisa da Embrapa (responsáveis por cada uma das tecnologias selecionadas) e atuantes na Caatinga participaram de um processo interativo de validação e qualificação destas tecnologias, no qual algumas foram excluídas e novas foram elencadas para compor a plataforma.

\footnotetext{
${ }^{2}$ www.embrapa.br
} 
Neste processo, uma atenção especial foi dada na organização dos materiais de apoio que auxiliam o produtor a efetivamente conhecer e adotar a tecnologia em questão (informações e instruções técnicas registradas em diferentes tipos de mídias como cartilhas/publicações, vídeos e programas de áudio). Além deste, o atributo onde encontrar (unidade da Embrapa responsável ou parceiros comerciais) foi revisado e tabulado para identificar os campos importantes que podem facilitar o acesso a cada tecnologia (endereço, telefone de contato e web site).

\subsection{Censo Agropecuário}

Apesar das fragilidades impostas pelas restrições hídricas e da imprevisibilidade do regime climático, a produção agropecuária da Caatinga é extremamente diversa. Esta diversidade é expressa tanto em variedade e volume produzido como nas diferentes realidades onde as atividades agropecuárias são desenvolvidas. As especificidades regionais, resultado de combinações únicas entre características naturais e sociais, influenciam diretamente as formas de se produzir no campo. Compreender os perfis produtivos da Caatinga é essencial para o planejamento e a adequada execução de políticas públicas de combate à desertificação, dado que o processo de degradação da terra se relaciona diretamente com os resultados da produção agropecuária.

Nesta plataforma, a análise deste perfil produtivo foi baseada na sistematização e qualificação de dados do Censo Agropecuário ${ }^{3}$. Coordenado e executado pelo Instituto Brasileiro de Geografia e Estatística (IBGE), em períodos decenais, o censo agropecuário é uma das principais referências para levantamento de dados sociais, econômicos e ambientais sobre o mundo rural brasileiro, e sua pesquisa mais recente foi realizada entre os anos de 2016 e 2017.Trata-se do único levantamento em que a totalidade dos produtores rurais é inserida na amostra, sendo a pesquisa de maior amplitude em termos de conhecimento de características das áreas rurais e da produção agropecuária existente em todo o território nacional.

O Censo é centrado no conceito de estabelecimento agropecuário, adotado como unidade básica para obtenção, caracterização e agregação de dados para divulgação. Diferentemente do imóvel rural, o estabelecimento agropecuário é definido como toda unidade de produção/exploração dedicada, total ou parcialmente, a atividades agropecuárias, florestais ou aquícolas, independente de seu tamanho, de sua forma jurídica ou de sua localização, com sua produção destinada à comercialização ou à subsistência [IBGE 2019]. Este conceito admite unidades de produção sem área associada (caso de apicultores ou pescadores, por exemplo).

Foram utilizados dois conjuntos de variáveis do Censo Agropecuário, com finalidades distintas na caracterização dos perfis agropecuários da Caatinga. O primeiro deles apresenta um quadro territorial das principais atividades agropecuárias da região, em termos econômicos e de uso da terra, a partir de duas variáveis e suas categorias específicas: valor da produção e utilização das terras. Enquanto o valor de produção representa os recursos financeiros gerados a partir da produção e sua comercialização, a utilização das terras apresenta a distribuição do uso das terras em diferentes atividades no interior dos estabelecimentos, considerando inclusive áreas destinadas à preservação ambiental.

\footnotetext{
${ }^{3}$ https://sidra.ibge.gov.br/pesquisa/censo-agropecuario/censo-agropecuario-2017
} 
O segundo conjunto apresenta indicadores mais específicos, a partir da seleção de produtos agropecuários. O objetivo é evidenciar as dinâmicas inerentes a cada cadeia produtiva e sua representatividade no bioma a partir de quatro variáveis que caracterizam o estabelecimento: enquadramento como agricultura familiar, tamanho de propriedade, condição legal e grupo de atividade econômica (principal). Estas características permitem o diagnóstico detalhado dos estabelecimentos agropecuários e identificação de perfis para políticas públicas, oportunidades de fomento e disseminação tecnológica.

\section{Resultados}

A plataforma está disponível para acesso público no portal da Embrapa ${ }^{4}$ e possui dois pontos de entrada: Tecnologias para prevenção e combate à desertificação e Perfil da agricultura e da pecuária regional. Visando viabilizar o acesso à plataforma em múltiplos dispositivos, todos os elementos visuais foram customizados para apresentação adequada na perspectiva mobile, favorecendo e ampliando o alcance do público alvo que pode fazer uso da ferramenta. As próximas seções descrevem os aspectos tecnológicos que embasam a plataforma e os resultados em cada um dos pontos de entrada.

\subsection{Aspectos Tecnológicos}

Os dados que embasam a plataforma foram organizados em um banco de dados relacional espacial (PostgreSQL + PostGIS). O diagrama de dados foi elaborado visando a normalização e consistência dos atributos e tendo em vista generalizar as particularidades diante dos seletores previstos para navegação na plataforma.

Os painéis interativos foram construídos utilizando-se o software de visualização de dados Tableau ${ }^{5}$. Conceitualmente, o Tableau categoriza os atributos em dois grupos: dimensões e medidas. Dimensões são valores qualitativos usados para categorização. Medidas correspondem a valores numéricos e quantitativos, passíveis de agregação (soma ou média, por exemplo). Os dados espaciais foram materializados como mapas e os demais atributos foram acomodados em diferentes apresentações visuais, de acordo com a natureza e classes de valores. Buscou-se a representação que melhor comportasse a maior parte dos casos. Os painéis forma publicados na nuvem pública do Tableau, onde os dados estão disponíveis para download em diferentes formatos.

Para a estruturação do conteúdo, foram construídas páginas web (HTML/CSS e JavaScript) que incorporam os painéis publicados. Através da API JavaScript do Tableau, os painéis podem ser acessados e filtrados de forma integrada com outros conteúdos descritivos, possibilitando uma maior interação. As páginas resultantes foram publicadas no gerenciador de conteúdos Liferay (ambiente corporativo da Embrapa), materializando a plataforma.

\subsection{Tecnologias para prevenção e combate à desertificação}

A Figura 1 apresenta o painel publicado junto à página Tecnologias para prevenção e combate à desertificação. As tecnologias foram indexadas por 8 categorias (de forma não unívoca): Conservação da Água e do Solo, Sistemas Integrados, Manejo Vegetal, Manejo Animal, Cultivares, Insumos e Implementos, Gestão e Serviços, Agroindústria.

\footnotetext{
${ }^{4}$ www.embrapa.br/bioma-caatinga/desertificacao/

${ }^{5}$ www.tableau.com
} 
Estas categorias são utilizadas na navegação da plataforma conforme mostrado no topo Figura 1. Com exceção das duas primeiras categorias, as demais possuem um segundo seletor de navegação referente ao produto agropecuário ao qual esta tecnologia se associa no processo produtivo.
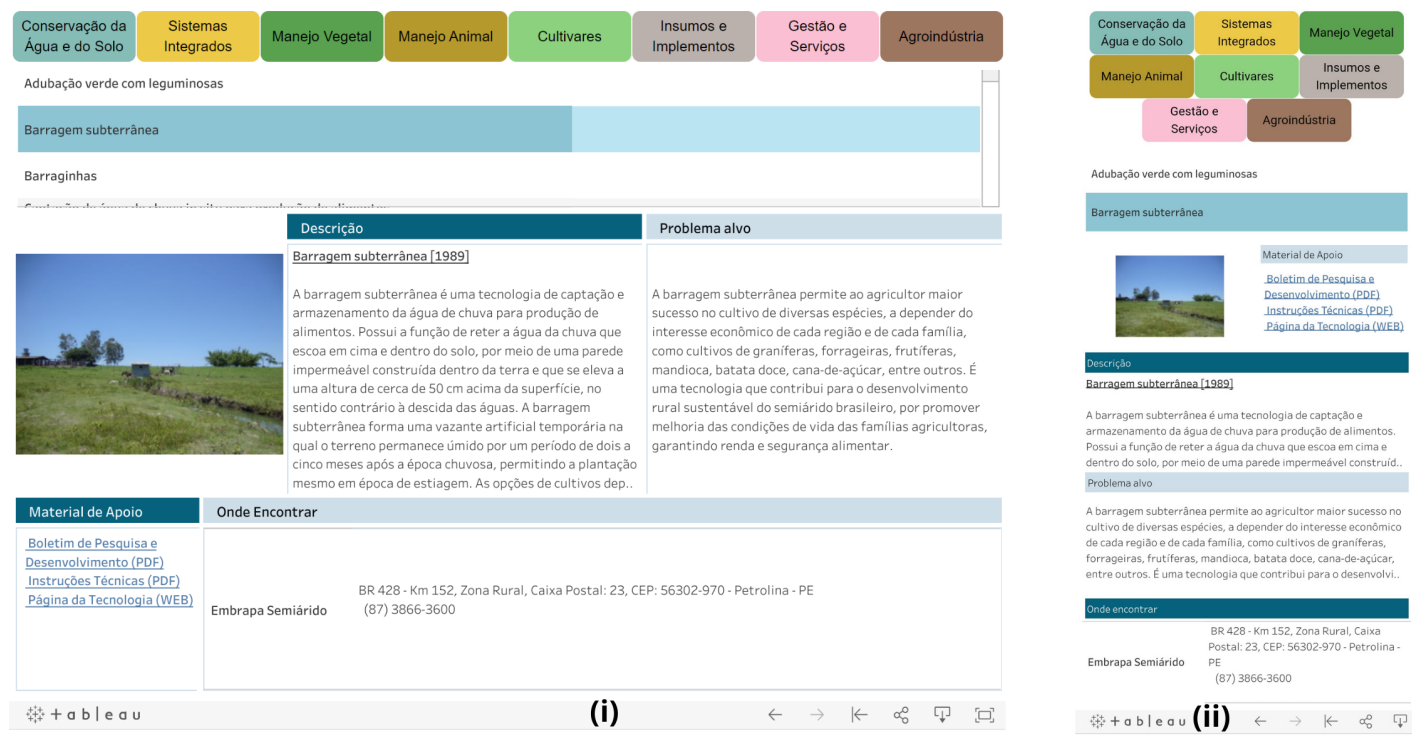

Figura 1. Painel de Tecnologias da Embrapa: (i) Desktop e (ii) Mobile

Como pode ser visto na Figura 1 item (i), a listagem de tecnologias é mostrada em uma tabela com rolagem, logo abaixo dos botões para seleção de categoria. Para uma tecnologia selecionada (na figura, Barragem subterrânea), é exibida uma imagem representativa da tecnologia, seguida horizontalmente de sua descrição e problema alvo. Abaixo, são mostrados os materiais de apoio disponíveis (um boletim de pesquisa e desenvolvimento, um folder de instruções técnicas e a página da tecnologia na web) e onde é possível encontra-la (Embrapa Semiárido). O clique neste último campo encaminha ao site quando disponível (neste caso, o portal institucional da Embrapa Semiárido).

\subsection{Perfil da agricultura e da pecuária regional}

A Figura 2 apresenta o primeiro painel publicado junto à página Perfil da agricultura e da pecuária regional. Ele apresenta dois aspectos: (a) as principais atividades econômicas da Caatinga em termos do valor gerado pela produção agropecuária (gráfico em barras na cor verde, na parte superior); e (b) a distribuição de utilização das terras nos estabelecimentos agropecuários da região (gráfico em barras roxas, na parte inferior). Além da apresentação gráfica, estes dados podem ser explorados de acordo com sua dispersão espacial (mapa em gradiente de cores terrosas, à esquerda do painel). O seletor no topo do mapa permite escolher a variável a ser espacializada no mapa.

É possível interagir com os conteúdos por meio da seleção de componentes em qualquer um dos gráficos (uma barra) ou do mapa (uma microrregião, por exemplo). A partir de uma interação, o painel se recalcula para apresentar as informações da seleção realizada. Ao selecionar uma microrregião no mapa de utilização das terras, por exem- 

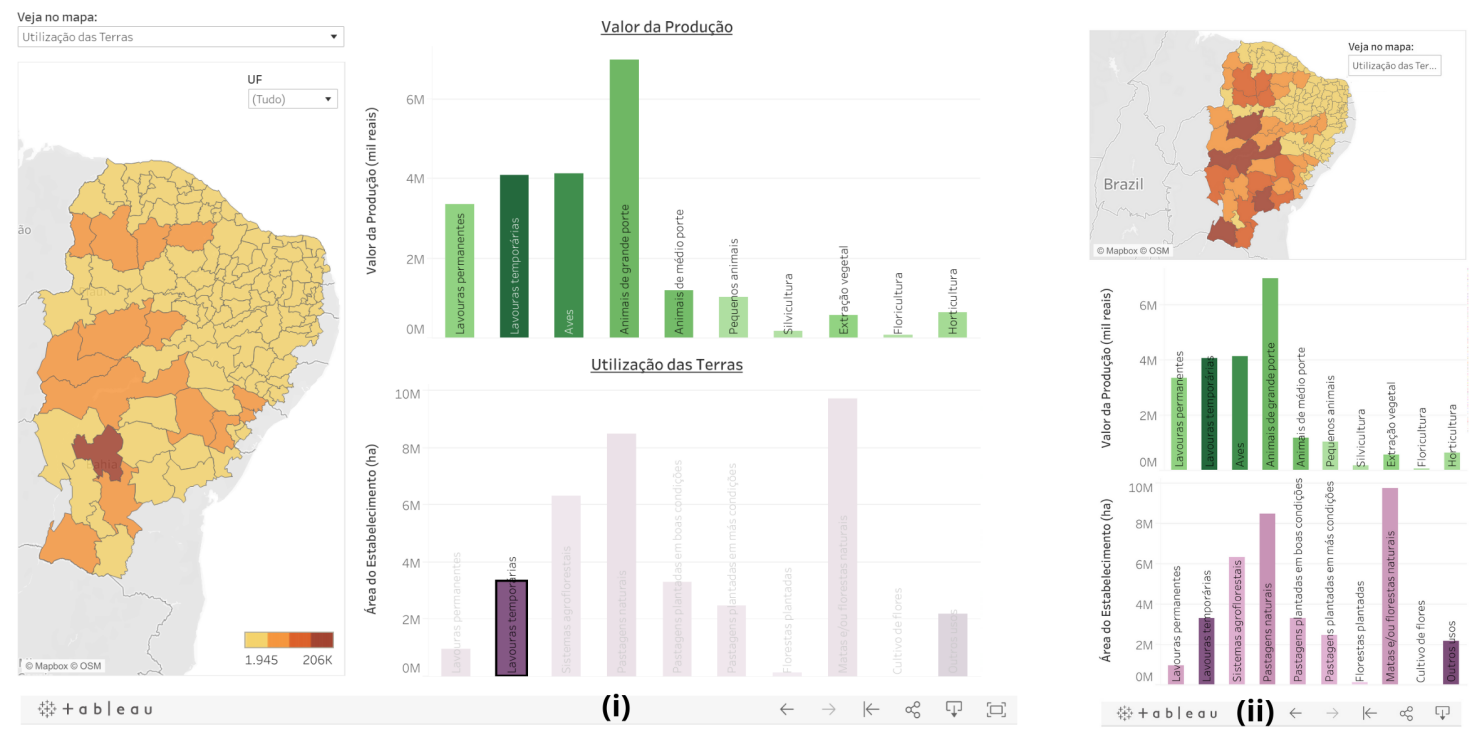

Figura 2. Dados Gerais do Censo Agropecuário na Caatinga: (i) Desktop e (ii) Mobile

plo, o gráfico correspondente irá apresentar distribuição das classes de utilização da terra apenas da microrregião selecionada.

O segundo painel, mostrado na Figura 3, apresenta uma visão dos estabelecimentos a partir dos produtos agropecuários. Os produtos foram organizados em 9 grupos, mostrados no topo do painel: Agroindústria, Aquicultura, Extração Vegetal, Lavoura Permanente, Lavoura Temporária, Leite de Vaca, Pecuária, Silvicultura e Total (representando todos os produtos). A partir da seleção de um grupo, é possível selecionar o produto e variável de interesse. De maneira geral, foram disponibilizadas duas variáveis: o número de estabelecimentos agropecuários e o volume produzido, que pode ser a quantidade em toneladas para lavouras, o número de caixas de colmeias para apicultura ou a quantidade de cabeças para pecuária.

O painel mostra, além da visão espacial dos dados (mapa à direita do painel, em gradiente de tons verdes e amarelos), quatro aspectos de caracterização: (a) enquadramento como agricultura familiar (em gráfico de pizza, à esquerda superior); (b) faixas de tamanho das propriedades (em gráfico de barras azuis, ao centro); (c) grupo de atividade econômica principal da propriedade (em mapa de árvore, à esquerda inferior); e (d) condição do produtor em relação à terra (em formato tabular, centralizado). Este painel tem um comportamento análogo ao anterior - a partir de seleção de uma microrregião no mapa (ou um conjunto de microrregiões), o painel se recalcula para apresentar as informações detalhadas de acordo com a seleção realizada.

A integração com o componente espacial nestes painéis permite obter tanto a visão geral do bioma como também o perfil detalhado de um estado, uma área de interesse ou uma microrregião. Isto permite, de forma concomitante, uma análise do perfil de variáveis com a distribuição territorial, evidenciando especificidades regionais produtivas, como padrões de uso e ocupação da terra ou presença da agricultura familiar na região. 


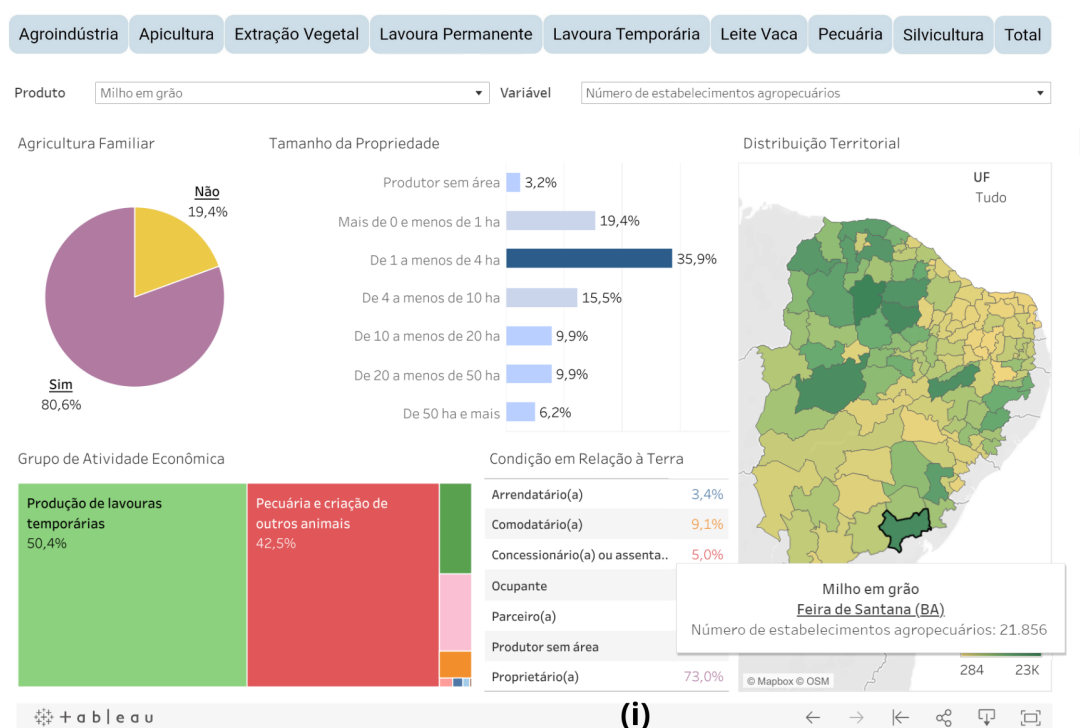

(i)

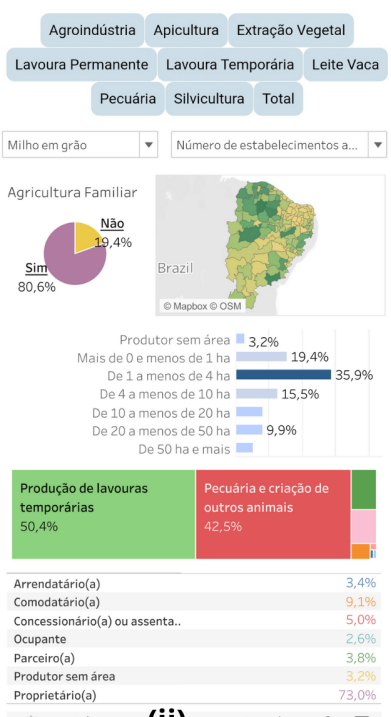

(ii)

Figura 3. Perfil da agricultura e da pecuária da Caatinga por produto: (i) Desktop e (ii) Mobile

\section{Contribuições e Trabalhos Futuros}

Após um vasto trabalho de filtragem e organização dos dados da Caatinga, foi possível construir uma base de dados espacial que agrega diferentes aspectos do bioma e, a partir dela, elaborar um conjunto de painéis interativos para exploração e análise dos dados. Os conteúdos presentes na plataforma por meio dos painéis permitem visualizar tendências sobre as principais características territoriais da agropecuária da Caatinga. Assim, essa visão é complementar à disponibilização das tecnologias da Embrapa no tema da prevenção e combate à desertificação na medida em que a utilização desses ativos deve ser necessariamente ancorada em contextos territoriais adequados à sua adoção.

A integração das informações, no âmbito das tecnologias e das características produtivas das regiões, amplia as possibilidades de associação entre os potenciais oferecidos por uma solução tecnológica ou por um conjunto de soluções frente às demandas e as características existentes em cada local, em diferentes realidades sociais, econômicas e ambientais. Quanto maior o conhecimento acerca das diferentes realidades geográficas associadas à desertificação, maior é a capacidade de planejamento e execução de políticas públicas assertivas, de promover o desenvolvimento agropecuário sustentável e de propor ações diferenciadas. Espera-se que esta visão espacial possa fornecer subsídios à elaboração de ações público/privadas no que se refere a este tema.

A plataforma está atualmente em fase de validação pelo MMA e parceiros da extensão rural. Os próximos passos abrangem a verificação da adequabilidade das apresentações visuais propostas (tipos de gráficos e adequações de cores e rótulos) e a estruturação de um processo para atualização contínua dos dados das tecnologias da Embrapa, dada a dinâmica necessária para evitar que a plataforma se torne obsoleta. 


\section{Referências}

Cowie, A. L., Orr, B. J., Castillo Sanchez, V. M., Chasek, P., Crossman, N. D., Erlewein, A., Louwagie, G., Maron, M., Metternicht, G. I., Minelli, S., Tengberg, A. E., Walter, S., e Welton, S. (2018). Land in balance: The scientific conceptual framework for land degradation neutrality. Environmental Science \& Policy, 79:25-35.

Critchley, W., Harari, N., e Mekdaschi-Studer, R. (2021). Restoring Life to the Land: The Role of Sustainable Land Management in Ecosystem Restoration. UNCCD and WOCAT.

da Silva, J. P., Guarneri, H., Arenas, F. C., de Paula, E. V., e Camboim, S. P. (2018). Uso de um Dashboard Geoespacial como ferramenta de suporte para o diagnóstico socioeconômico e ambiental da Reserva Biológica Bom Jesus - Litoral do Paraná. In Proceedings XIX GEOINFO, pages 152-157, Campina Grande, PB.

IBGE (2019). Censo Agropecuário 2017: Resultados Definitivos. Instituto Brasileiro de Geografia e Estatística, Rio de Janeiro.

PAN-Brasil (2004). Programa de ação nacional de combate a desertificação em mitigação dos efeitos da seca. Relatório Técnico, Ministério do Meio Ambiente/Secretaria de Recursos Hidrícos, Brasília.

Penteado-Filho, R. C. (2010). Diagnóstico e propostas de soluções para a transferência de tecnologia numa empresa pública de pesquisa agropecuária: o caso Embrapa. Prisma.com (Portugual), 11:86-102.

Pinto, D. M., Magalhães, L. A., Takemura, C. M., Farias, A. R., de Almeida, B. T., dos Santos Brandão, V. V., e da Conceição, A. R. (2019). Metodologia para espacialização das soluções tecnológicas da Embrapa. Circular Técnica 34, Embrapa Territorial, Campinas.

UNCCD (1994). United Nations Convention to Combat Desertification in Those Countries Experiencing Serious Drought and/or Desertification Particularly in Africa: Text with Annexes. United Nations Convention to Combat Desertification. UNEP, Nairobi.

UNCCD (2019). Land Degradation Neutrality for Biodiversity Conservation. United Nations Convention to Combat Desertification. Briefing Note. Bonn, German. 